\title{
Planetary Nebulae in the UWISH2 Galactic Plane survey
}

\author{
T. M. Gledhill ${ }^{1}$ and D. Froebrich ${ }^{2}$ \\ ${ }^{1}$ Centre for Astrophysics Research, University of Hertfordshire, \\ College Lane, Hatfield, AL10 9AB, UK \\ email: t.gledhill@herts.ac.uk \\ ${ }^{2}$ Centre for Astrophysics \& Planetary Science, The University of Kent, \\ Canterbury, CT2 7NH, UK
}

\begin{abstract}
UWISH2 is the first unbiassed imaging survey of $\mathrm{v}=1-0 \mathrm{~S}(1)$ molecular hydrogen emission $(\lambda=2.122 \mu \mathrm{m})$ in the northern Galactic Plane. Here we discuss 284 extended emission line objects which we consider to be candidate planetary or pre-planetary nebulae. Some are clearly associated with known PN, but the majority $(60 \%)$ have no previous detection. We have classified the objects according to morphology and find $53 \%$ are bipolar with half of these being new detections. The remaining objects are mostly elliptical/round (35\%), fainter than the median sample flux $\left(4.4 \times 10^{-17} \mathrm{~W} \mathrm{~m}^{-2}\right)$ and previously undetected $(74 \%)$.
\end{abstract}

Keywords. planetary nebulae: general, surveys, stars: AGB and post-AGB, stars: evolution

\section{Introduction}

The number of known planetary nebulae (PN) in the Galaxy is in excess of 3,400 (e.g. Frew et al. 2016 and these Proceedings). Most of these discoveries were made at optical wavelengths, with a large number coming from wide-field Galactic Plane (GP) surveys in $\mathrm{H} \alpha$, such as IPHAS (Sabin et al. 2014) and SHS (Parker et al. 2006; Miszalski et al. 2008). However, the population of optically-obscured PN remains largely unexplored, and a significant number of objects may be expected to lie hidden behind dust clouds in the GP. Neglecting these optically-invisible objects will lead to an underestimate of the total number of PN as well as potential biassing of the population to the brighter and/or closer (and therefore possibly atypical) objects. The need to move the emphasis to detection at longer wavelengths has already been highlighted, for example by establishing mid-IR diagnostics based on Spitzer IRAC colours and mid-IR-to-radio flux ratios (Cohen et al. 2011; Parker et al. 2012).

In the near-IR $K$ band (between 2 and $2.5 \mu \mathrm{m}$ ) molecular hydrogen $\left(\mathrm{H}_{2}\right)$, excited by UV photons or by collisions, can produce a characteristic ro-vibrational spectrum with the $\mathrm{v}=1-0 \mathrm{~S}(1)$ line at $2.122 \mu \mathrm{m}$ being most prominent. This line has often been used as a signpost of photodissociation regions and of gas heated in shocks, and is a particularly useful tracer of PN as well as their non-ionised post-AGB predecessors, pre-PN or PPN. A number of targeted $\mathrm{H}_{2}$ observations of $\mathrm{PN}$ and $\mathrm{PPN}$ samples have been made over the years (e.g. Kastner et al. 1996; Kelly \& Hrivnak 2004) but until now there has been no systematic $\mathrm{H}_{2}$ survey of the GP from which to identify previously unknown PN and PPN candidates.

We have recently completed the first unbiassed imaging survey for $\mathrm{H}_{2} \mathrm{v}=1-0 \mathrm{~S}(1)$ emission in the northern GP using the Wide Field Camera (WFCAM) at the 3.8-m UK Infrared Telescope. The UWISH2 survey (Froebrich et al. 2011) covers a contiguous area of the Plane, typically with latitudes between $\pm 1.5^{\circ}$, and extending from $l=357^{\circ}$ 


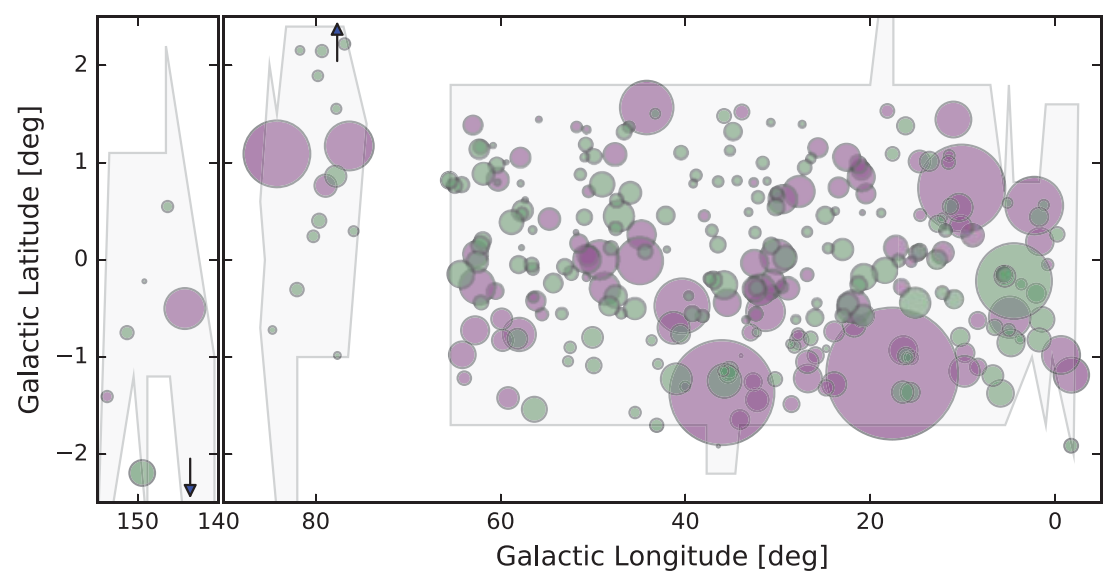

Figure 1. The distribution of $\mathrm{H}_{2}$ emission line objects flagged as PN or PN candidates. Circle size is proportional to area on the sky. The two arrows point to objects lying off the diagram. See text for further details.

through the Galactic Centre to $l=65^{\circ}$. With the addition of smaller fields centred on the Cygnus and Cassiopeia/Auriga star-forming regions, the survey includes a total area of 287 square degrees. A large number (over 32,000) of individual extended $\mathrm{H}_{2}$ features were identified, leading to 284 unique groups of features classified as candidate PN or PPN (Froebrich et al. 2015). Some of these detections are clearly associated with known PN whereas others have no previously-recorded affiliation. The number of candidate $\mathrm{PN}$ is likely to increase, as faint $\mathrm{H}_{2}$ detections initially classed as likely HII regions or "unknown", are reassessed.

\section{Distribution, morphology and $\mathbf{H} \alpha$ emission}

The distribution of the $284 \mathrm{H}_{2}$ objects is shown in Fig. 1 with the polygonal outlines indicating the footprint formed by the survey tiles and including the two smaller areas in Cygnus $\left(74^{\circ}<l<86^{\circ}\right)$ and Auriga $\left(140^{\circ}<l<155^{\circ}\right)$. The circle sizes are scaled linearly according to the extent of emission on the sky, and we divide objects into previously known PN (purple) and those which appear to be new detections of candidate (P)PN (green). The latter number 170 , or $60 \%$ of the total, with many seen to be small (and faint) relative to the known objects.

We have classified the sample according to $\mathrm{H}_{2}$ morphology using the "ERBIAS" system (Parker et al. 2006) with results summarized in Fig. 2. The majority of objects (151 or $53 \%$ ) appear bipolar with 75 of these being new detections. The remaining objects are mostly elliptical or round (aspect ratio less than 1.05) totalling 100 or (35\%) and of these $74 \%$ are new detections.

The UWISH2 survey area is included within two H $\alpha$ surveys: the SHS (Parker et al. 2006) covering the Galactic Centre and inner plane to longitudes of $l \approx 35^{\circ}$ and the IPHAS (Drew et al. 2005) which extends outwards along the plane from $l \approx 29^{\circ}$. Any point in UWISH2 therefore lies within one or both of these surveys which allows us to search for $\mathrm{H} \alpha$ emission in our $\mathrm{H}_{2}$ targets and to compare morphologies. Fig. 2 also shows the proportion of objects present/absent from the $\mathrm{H} \alpha$ surveys. The majority of $\mathrm{B}$ (bipolar) $\mathrm{PN}$ with detectable $\mathrm{H}_{2}$ emission also emit in $\mathrm{H} \alpha$ although $69 / 151$ (46\%) do not. In the remaining categories (ERAIS), objects without $\mathrm{H} \alpha$ outnumber those with, 


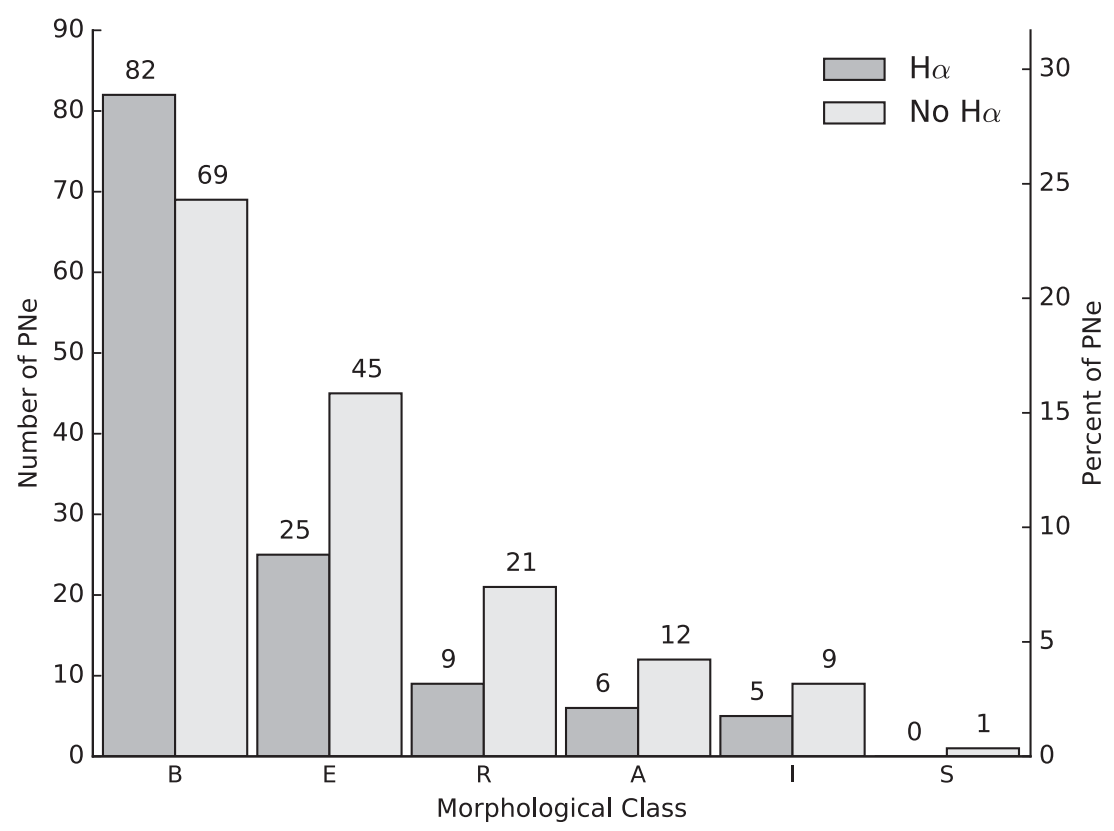

Figure 2. ERBIAS classification of UWISH2 objects and presence/absence of $\mathrm{H} \alpha$ emission.

by approximately 2:1. Of the 170 new candidate (P)PN in UWISH2, $142(84 \%)$ are not seen in $\mathrm{H} \alpha$.

\section{Flux and surface brightness distribution}

The flux in the $\mathrm{v}=1-0 \mathrm{~S}(1)$ line is calculated for each object by summing the background-corrected emission within regions lying above the $5 \sigma$ pixel noise. More than one region may contribute to an object and the surface area (in square arcseconds) is then the sum of the area of each region (see Froebrich et al. 2015 for details and values). In Fig. 3 we show the distribution of both flux and surface brightness (i.e. flux divided by area) for our sample, divided according to the presence/absence of detectable $\mathrm{H} \alpha$ emission. The median flux is $438 \times 10^{-19} \mathrm{~W} \mathrm{~m}^{-2}$ with a significant proportion of faint objects and a long tail towards bright objects. This bright tail is made up mostly of known PN, with $\mathrm{H} \alpha$ emission (median $906 \times 10^{-19} \mathrm{~W} \mathrm{~m}^{-2}$ ), whereas the peak towards the faint end is almost exclusively due to objects without $\mathrm{H} \alpha$ emission (median $316 \times$ $10^{-19} \mathrm{~W} \mathrm{~m}^{-2}$ ). Hence UWISH2 is detecting a population of new and faint objects which are potentially optically-obscured PN. The surface brightness distribution, however, is very similar across the sample, regardless of $\mathrm{H} \alpha$ detection, and sharply peaked with a median value of $4 \times 10^{-19} \mathrm{~W} \mathrm{~m}^{-2} \operatorname{arcsec}^{-2}$. In other words, the faint objects are small, which is consistent with the idea of probing longer sightlines to more distant and reddened objects in the $K$-band. If we split the sample according to morphology then we find that the majority of objects fainter than the median flux are $\mathrm{E} / \mathrm{R}$; the median fluxes for "E/R" and "B" types are 262 and $664 \times 10^{-19} \mathrm{~W} \mathrm{~m}^{-2}$ respectively. To ascertain how many of these objects are obscured PN, PPN without ionized regions or other unrelated objects, will require further observations such as near-IR spectroscopy, which is in progress. 

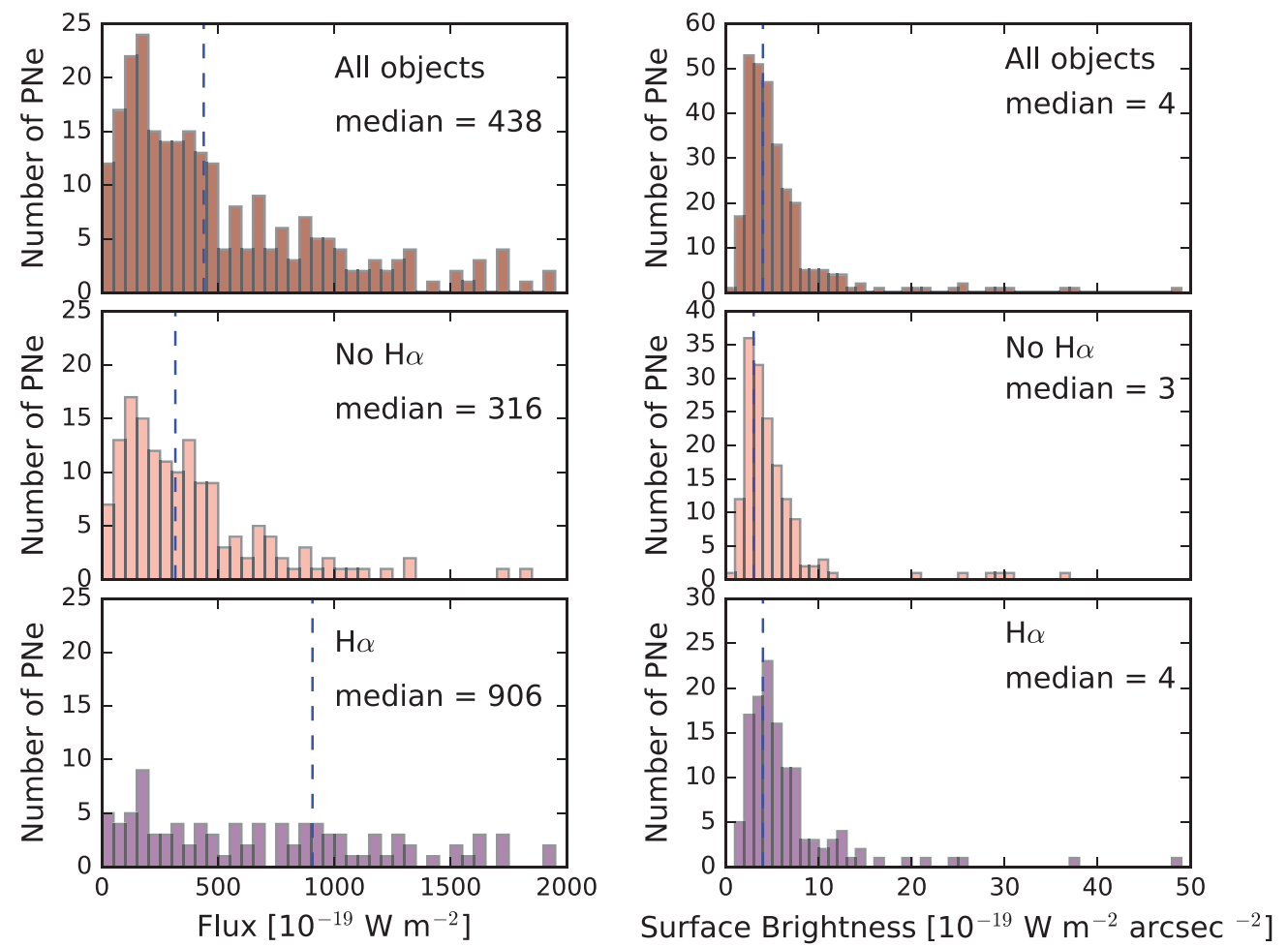

Figure 3. $v=1-0 \mathrm{~S}(1)$ emission line flux and surface brightness distributions.

\section{References}

Cohen, M., Parker, Q. A., Green A. J., Miszalski, B., Frew, D., \& Murphy, T. 2011, MNRAS, 413,514

Drew, J., et al. 2005, MNRAS, 362, 753

Frew, David J., Parker, Q. A., \& Bojicic, I. S. 2016, MNRAS, 455, 1459

Froebrich, D., et al. 2011, MNRAS, 413, 480

Froebrich, D., et al. 2015, MNRAS, 454, 4679

Kastner, J. H., Weintraub, D. A., Gatley, I., Merrill, K. M., \& Probst, R. G. 1996, ApJ, 462, 777

Kelly, D. M. \& Hrivnak, B. J., 2005, ApJ, 629, 1040

Miszalski, B., Parker, Q. A., Acker, A., Birkby, J. L., Frew, D. J., \& Kovacevic, A. 2008, MNRAS, 384,525

Parker, Q. A., et al. 2006, MNRAS, 373, 79

Parker, Q. A., Cohen, M., Stupar, M., Frew, D. J., Green, A. J., Bojicic, I., Guzman-Ramirez, L., Sabin, L., \& Vogt, F. 2012, MNRAS, 427, 3016

Sabin, L., et al. 2014, MNRAS, 443, 3388

\section{Discussion}

SCHOENBERNER: It would be very important to place all these objects in the HR diagram! So you have to look for the central stars, too.

DE MARCO: Why such a large fraction of bipolars?

GLEDHILL: Some objects are distant. The bipolar structure stands out in H2 while a possible elliptical structure may be too faint at larger distances. 